\title{
Dipole strength effects on the polymorphism in smectic A mesophases
}

\author{
Roberto Berardi, ${ }^{a}$ Silvia Orlandi, ${ }^{a b}$ Demetri J. Photinos, ${ }^{b c}$ Alexandros G. Vanakaras ${ }^{a c}$ \\ and Claudio Zannoni ${ }^{a}$ \\ a Dipartimento di Chimica Fisica ed Inorganica, and INSTM Unit, Università di Bologna, \\ Viale Risorgimento 4, 40136 Bologna, Italy \\ ${ }^{b}$ Department of Physics, University of Patras, Patras 26500, Greece \\ c Department of Materials Science, University of Patras, Patras 26500, Greece
}

\author{
Received 30th July 2001, Accepted 25th October 2001 \\ First published as an Advance Article on the web 23rd January 2002
}

\begin{abstract}
We study the effect of molecular dipole strength on the polymorphism of smectic A liquid crystals using computer simulations and density functional theory. We find, for a system of polar Gay-Berne particles with an off-centre axial dipole, a change in molecular organisation from non-interdigitated to interdigitated $\left(\mathrm{S}_{\mathrm{A}_{\mathrm{d}}}\right)$ structure.
\end{abstract}

\section{Introduction}

In a simple classification of liquid crystals, nematics have longrange orientational order, while smectic phases also have additional translational order corresponding to a layered molecular organisation. The variety of smectics is, however, very wide ${ }^{1,2}$ and comprises a number of phases of increasing complexity, from the most intuitive instance of molecules perpendicular to each layer and positionally disordered $\left(\mathrm{S}_{\mathrm{A}}\right)$, to the case of molecules that are tilted and/or have some degree of positional order inside the layers $\left(\mathrm{S}_{\mathrm{C}}, \mathrm{S}_{\mathrm{B}}\right.$, etc. $)$. $^{1-4}$

An additional and perhaps more surprising polymorphism is that found in smectic A phases formed by strongly polar molecules, in particular rod-like mesogens with a permanent dipole near one end of the molecule and a single chain at the other end. ${ }^{2,4-8}$ In this case the layer spacing as measured by $\mathrm{X}$ rays varies between one $\left(\mathrm{S}_{\mathrm{A}_{1}}\right)$ and two $\left(\mathrm{S}_{\mathrm{A}_{2}}\right)$ full molecular lengths, with intermediate values in interdigitated $S_{\mathrm{A}_{d}}$ phases, while the dipoles themselves can arrange in different ways. Thus a double layer with antiferroelectric order of dipoles is found in $\mathrm{S}_{\mathrm{A}_{2}},{ }^{3,6}$ a striped dipolar domain structure in $\mathrm{S}_{\tilde{\mathrm{A}}},{ }^{3,9}$ while a compensation of the dipoles seems to be associated with the interdigitated structure. ${ }^{3}$

Another indication of the delicate balance of interactions in these smectics is the observation, in some cases, of re-entrant nematic phases on further cooling down an already formed smectic. ${ }^{5}$

Disentangling the effect of different molecular interactions such as steric repulsion, dispersive attraction and dipolar forces (not to mention conformational and chain-length-related effects) in real mesogens is not easy, given the complex nature of the molecules of interest or of their mixtures, and there is a need for simple model systems that could help in understanding the role of the various contributions.

On an intuitive basis, it would seem reasonable that the position and strength of the mesogen dipole moment is important in this respect. However, this is not so obvious and not so easy to demonstrate, and there is at least one case ${ }^{10}$ where, in a computer simulation of a system of hard polar spherocylinders, only a single monolayer $\mathrm{S}_{\mathrm{A}}$ phase was obtained irrespective of the location and strength of the axial dipole. A number of other simulations of polar hard particles $^{10,11}$ and Gay-Berne (GB) systems ${ }^{12-15}$ have appeared.
Central $^{12,13}$ and terminal ${ }^{12,14}$ axial dipoles of different strength have been studied. The dipoles are generally seen to influence the nematic-smectic more than the nematic-isotropic transition. Without reviewing all the different findings, we just mention that $S_{A}$ and $S_{B}$ phases have been observed, but not the variety of experimentally observed smectic phases.

In this paper we wish to investigate the extent to which the polymorphism is affected by the strength of a longitudinal dipole placed at a suitable non-central position along the molecular axis. To do this we choose to simulate a system of ellipsoidal particles with length to width ratio 3:1 interacting via an attractive-repulsive Gay-Berne (GB) potential with an added longitudinal point dipole as detailed later on in Section 2.

We shall present both extensive Monte Carlo (MC) simulations (Section 3) and a theoretical analysis (Section 4), and we discuss our findings in Section 5.

\section{Model and computer simulations}

We consider a system of elongated uniaxial ellipsoidal particles of length $\sigma_{\mathrm{e}}$ and breadth $\sigma_{\mathrm{s}}$ with an embedded axial point dipole at a selected off-centre position on the axis, and we study the influence of the strength of this molecular dipole on the overall mesophase structure.

The dimensionless pair potential $U^{*}(i, j)=U(i, j) / \varepsilon_{\mathrm{s}}$ that we adopt is the sum of a Gay-Berne ${ }^{16,17}$ and a dipole-dipole term:

$$
U^{*}(i, j)=U^{\mathrm{GB} *}(i, j)+U^{\mathrm{d} *}(i, j),
$$

where the Gay-Berne part contains the anisotropic attractiverepulsive contribution between the two particles with orientation vectors $\hat{\boldsymbol{u}}_{i}, \hat{\boldsymbol{u}}_{j}$ and center-centre separation $\boldsymbol{r}_{i j}=r_{i j} \hat{\boldsymbol{r}}_{i j}$, and where $\varepsilon_{\mathrm{s}}$ is the energy unit. Molecular orientations are defined with respect to the principal axes of particles $i, j$, namely $\hat{\boldsymbol{u}}_{i} \equiv \hat{\boldsymbol{z}}_{i}$ and $\hat{\boldsymbol{u}}_{j} \equiv \hat{\boldsymbol{z}}_{j}$ (we use a 'cap' to indicate a unit vector):

$$
\begin{aligned}
U^{\mathrm{GB} *}(i, j)= & 4 \varepsilon\left(\hat{\boldsymbol{r}}_{i j}, \hat{\boldsymbol{u}}_{i}, \hat{\boldsymbol{u}}_{j}\right)\left\{\left[\frac{\sigma_{\mathrm{s}}}{r_{i j}-\sigma\left(\hat{\boldsymbol{r}}_{i j}, \hat{\boldsymbol{u}}_{i}, \hat{\boldsymbol{u}}_{j}\right)+\sigma_{\mathrm{s}}}\right]^{12}\right. \\
& \left.-\left[\frac{\sigma_{\mathrm{s}}}{r_{i j}-\sigma\left(\hat{\boldsymbol{r}}_{i j}, \hat{\boldsymbol{u}}_{i}, \hat{\boldsymbol{u}}_{j}\right)+\sigma_{\mathrm{s}}}\right]^{6}\right\} .
\end{aligned}
$$


Here $\varepsilon\left(\hat{\boldsymbol{r}}_{i j}, \hat{\boldsymbol{u}}_{i}, \hat{\boldsymbol{u}}_{j}\right)$ and $\sigma\left(\hat{\boldsymbol{r}}_{i j}, \hat{\boldsymbol{u}}_{i}, \hat{\boldsymbol{u}}_{j}\right)$ are the strength of interaction and contact distance whose explicit form is given elsewhere. ${ }^{16,17}$ We employ shape anisotropy $\sigma_{\mathrm{e}} / \sigma_{\mathrm{s}}=3$, a ratio of side-to-side to end-to-end interaction $\varepsilon_{\mathrm{s}} / \varepsilon_{\mathrm{e}}=5$, and GB exponential coefficients $\mu=1, v=3$, corresponding to the parametrisation introduced elsewhere. ${ }^{17}$ The cutoff radius adopted is $R_{\mathrm{c}}^{\mathrm{GB}}=4.0 \sigma_{\mathrm{s}}$. In the absence of dipoles this GB model, at density $\rho^{*} \equiv N / V^{*}=\sigma_{\mathrm{s}}{ }^{3} N / V=0.3$, has a transition from isotropic to nematic phase at a scaled temperature $\left(T^{*}=k_{\mathrm{B}} T / \varepsilon_{\mathrm{s}}\right) T_{\mathrm{NI}}{ }^{*} \approx 3.55$ and then becomes smectic at $T_{\mathrm{NS}}{ }^{*} \approx 2.4$.

The dipolar energy is given by the standard electrostatic expression:

$$
U^{\mathrm{d} *}(i, j)=\frac{\mu_{i}^{*} \mu_{j}^{*}}{r_{\mathrm{d}}^{* 3}}\left[\hat{\boldsymbol{z}}_{i} \cdot \hat{\boldsymbol{z}}_{j}-3\left(\hat{\boldsymbol{z}}_{i} \cdot \hat{\boldsymbol{r}}_{\mathrm{d}}\right)\left(\hat{\boldsymbol{z}}_{j} \cdot \hat{\boldsymbol{r}}_{\mathrm{d}}\right)\right]
$$

where $\boldsymbol{r}_{\mathrm{d}} \equiv r_{\mathrm{d}} \hat{\boldsymbol{r}}_{\mathrm{d}}$ is the vector joining the two point dipoles at distance $r_{\mathrm{d}}=\sigma_{\mathrm{s}} r_{\mathrm{d}}{ }^{*}$. We consider an embedded electric point dipole oriented along the molecular axis, located at an intermediate distance between centre and end $\left(d^{*}=d / \sigma_{\mathrm{s}}=0.65\right)$ and dipole moments $\boldsymbol{\mu}_{i}^{*} \equiv \mu^{*} \hat{\boldsymbol{z}}_{i}, \boldsymbol{\mu}_{j}^{*} \equiv \mu^{*} \hat{\boldsymbol{z}}_{j}$, where $\mu^{*} \equiv\left(\mu^{2} / \varepsilon_{\mathrm{s}} \sigma_{\mathrm{s}}^{3}\right)^{1 / 2}$ is the dimensionless moment. This choice of dipole location ensures that a frustration is built into the system, as GB and dipolar interactions cannot be simultaneously optimised by a standard smectic layering. This in turn might be of use in trying to change the molecular organisation by changing the relative contribution of the dipolar term. The dipolar interaction has been computed using the reaction field (RF) method $^{18}$ with cutoff radius $R_{\mathrm{c}}{ }^{\mathrm{RF}}=6.0 \sigma_{\mathrm{s}}$ and dielectric constant of the surrounding medium $\varepsilon^{\mathrm{RF}}=1.5$, as in ref. 19. Various groups ${ }^{19-21}$ have shown that the RF approach is sufficiently accurate for liquid-crystal systems of the size as large as ours. As a further check we have also performed simulations with the Ewald summation method $\left(\alpha=4.8, k_{\max }=2\right.$, tin-foil boundary conditions ${ }^{12}$ ) for the case $\mu^{*}=2.0$, finding the same values for dipolar energies and order parameters as obtained from the RF simulations.

In the theoretical calculations the intermolecular potential is simplified by replacing the repulsive part of the Gay-Berne potential with a hard-body repulsive term when the molecules approach each other closer than the contact distance $\sigma\left(\hat{\boldsymbol{r}}_{i j}, \hat{\boldsymbol{u}}_{i}\right.$, $\hat{\boldsymbol{u}}_{j}$ ). With this approximation, the intermolecular potential can be written as

$$
U^{*}(i, j)=U^{\text {hard } *}(i, j)+U^{\text {soft* }}(i, j),
$$

where $U^{\text {hard* }}$ corresponds to the hard Gaussian overlap (HGO) model

$$
U^{\text {hard } *}(i, j)= \begin{cases}\infty & \text { if } r_{i j}<\sigma\left(\hat{\boldsymbol{r}}_{i j}, \hat{\boldsymbol{u}}_{i}, \hat{\boldsymbol{u}}_{j}\right), \\ 0 & \text { if } r_{i j} \geqslant \sigma\left(\hat{\boldsymbol{r}}_{i j}, \hat{\boldsymbol{u}}_{i}, \hat{\boldsymbol{u}}_{j}\right),\end{cases}
$$

and the second term $U^{\text {soft* }}$ collects the residual interactions, namely the remaining 'soft' repulsion and attraction of the Gay-Berne part as well the dipolar interactions,

$$
U^{\mathrm{soft} *}(i, j)= \begin{cases}0 & \text { if } r_{i j}<\sigma\left(\hat{\boldsymbol{r}}_{i j}, \hat{\boldsymbol{u}}_{i}, \hat{\boldsymbol{u}}_{j}\right), \\ U^{\mathrm{GB} *}(i, j)+U^{\mathrm{d} *}(i, j) & \text { if } r_{i j} \geqslant \sigma\left(\hat{\boldsymbol{r}}_{i j}, \hat{\boldsymbol{u}}_{i}, \hat{\boldsymbol{u}}_{j}\right) .\end{cases}
$$

Our MC sample consisted of $N=1000$ interacting particles as described in the present section at a dimensionless density $\rho^{*}=0.3$ and we have investigated several temperatures $T^{*}$ corresponding to nematic and smectic phases for the apolar systems. We have used canonical (constant volume and temperature, $N V T$ ) conditions as in ref. 17 and with cubic periodic boundary conditions.

The simulations were typically run in a cooling sequence with equilibration runs of $\approx 200$ kcycles and production runs of 100-300 kcycles. Observables for averaging and data analysis were accumulated during production runs sampling one configuration each 20. The MC evolution of configurations takes place via positional and orientational moves ${ }^{17}$ with the addition of attempted dipole flips to prevent locking in metastable states, ${ }^{12}$ a flip movle being a rotation of 180 degrees around the particle $x$ axis. The flip moves were randomly attempted with a $20 \%$ frequency compared to the $80 \%$ of the conventional translational-orientational moves.

\section{Results from simulations}

We plot in Fig. 1 our results for the average values of the dimensionless Gay-Berne energy per particle $\left\langle U^{\mathrm{GB} *}\right\rangle$, the dipolar energy $\left\langle U^{\mathrm{d} *}\right\rangle$ and the second-rank orientational order parameter $\left\langle P_{2}\right\rangle$, at different temperatures for the three dipole strengths.

The orientational order parameter $\left\langle P_{2}\right\rangle=\left\langle\left[3(\hat{\boldsymbol{u}} \cdot \hat{\boldsymbol{e}})^{2}-1\right] / 2\right\rangle$ was calculated as in, ref. 17 obtaining at each selected configuration the director $\hat{\boldsymbol{e}}$ from diagonalisation of the ordering matrix. ${ }^{22-24}$ Similarly, $\left\langle P_{1}\right\rangle=\langle\hat{\boldsymbol{u}} \cdot \hat{\boldsymbol{e}}\rangle$ was routinely calculated, but was always found to be zero within error, and thus it is not reported here.

All the systems studied present a nematic-isotropic (NI) and a nematic-smectic (NS) transition in the temperature range studied. The order parameter increases regularly with decreasing temperature, with no sign of re-entrance in all the cases studied.

We notice that switching on and increasing the strength of the dipole has a greater effect on the nematic-smectic transition, which is shifted to lower temperature. The temperature shift is, however, not monotonic with respect to dipolar strength, as we can see from Fig. 1. The nematic-isotropic transition is not affected much by the dipole, as was also found for the case of a central axial dipole for a GB system with a different parametrisation, ${ }^{13}$ while a significant shift to higher temperatures was instead found for the nematic-isotropic transition in strongly polar GB particles with a terminal dipole (from $T_{\mathrm{NI}}{ }^{*}=2.2$ to $T_{\mathrm{NI}}{ }^{*}=3$ on going from $\mu^{*}=0$ to $\mu^{*}=2$, ref. 14).

In this work we have concentrated on the smectic phases and on the types of polar organisation obtained. The limit between low-temperature smectic and solid phases is always difficult to assign; and here, to confirm the smectic, fluid, nature of the phases, we have calculated the mean-square displacements $l_{i}$ in the different directions. These are definitely non-negligible even at the lowest temperature studied, where they are not lower than $0.1 \sigma_{\mathrm{s}}$, to be compared with typical values of $\approx 0.3 \sigma_{\mathrm{s}}$ in the isotropic phase.

Inspection of the snapshots (Fig. 2) allows some interesting observations to be made concerning the molecular arrangement. The lowest dipole $\mu^{*}=1$ system is characterised by monolayer smectic, in which each layer contains approximately an equal number of molecules with dipole moments pointing 'up' and 'down' (shown in Fig. 2 as black and white arrows), along the director, so that the layers have no net polarisation. By contrast, the dipolar molecules with $\mu^{*}=1.5$ and $\mu^{*}=2$ tend to be arranged in such a way that the opposite dipoles in neighbouring molecules are located at a minimum distance, giving rise to the formation of interdigitated smectic $\mathrm{S}_{\mathrm{A}_{\mathrm{d}}}$ phases, with layer spacing $\sigma_{\mathrm{e}}<s_{\mathrm{d}}<2 \sigma_{\mathrm{e}}$. Corresponding to this pairing, the dipolar contribution to the energy goes from being essentially negligible for $\mu^{*}=1$ to slightly negative for $\mu^{*}=1.5$ and reaching $25 \%$ of the total energy for $\mu^{*}=2$ (Fig. 1).

In order to analyse in a quantitative way the molecular and dipolar organisation, we have computed the radial distribution functions $g_{0}(r)$ as

$$
g_{0}(r)=\frac{1}{4 \pi r^{2} \rho}\left\langle\delta\left(r-r_{12}\right)\right\rangle_{12},
$$

where $\langle\ldots\rangle_{12}$ represents an average with respect to all the molecular pairs, and the density along the director $g(z)$ as 

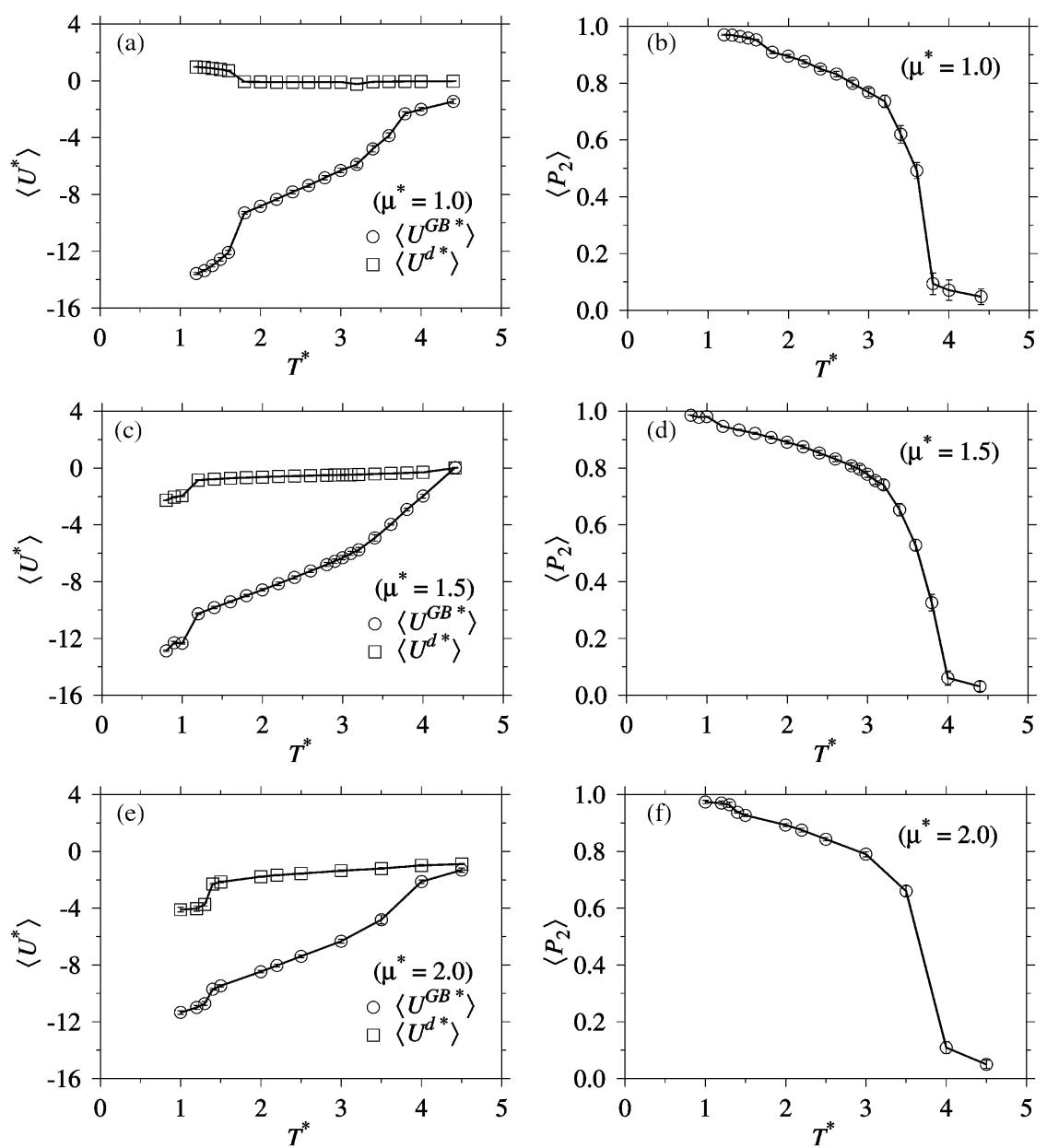

Fig. 1 Temperature dependence of the average dimensionless Gay-Berne $\left\langle U^{\mathrm{GB} *}\right\rangle$ and dipolar $\left\langle U^{\mathrm{d} *}\right\rangle$ energies per particle [panels (a), (c) and (e)] and of the second-rank orientational order parameter $\left\langle P_{2}\right\rangle$ [panels (b), (d) and (f)] for the three dipole strengths studied with the MC simulations described in the text.

$$
g(z)=\frac{1}{\pi R^{2} \rho}\left\langle\delta\left(z-z_{12}\right)\right\rangle_{12}
$$

which gives the probability for the intermolecular vector to have a projection $z$ along the director. Here $z_{12}=\boldsymbol{r}_{12} \cdot \hat{\boldsymbol{e}}$ is measured with respect to the director frame, $R$ is the radius of a cylindrical sampling region, and the averages were computed over all the molecular pairs. The density of dipoles along the director $g\left(z_{\mathrm{d}}\right)$ has been determined in a similar fashion using $z_{\mathrm{d}_{12}}=\boldsymbol{r}_{\mathrm{d}} \cdot \hat{\boldsymbol{e}}$.

We have also computed the spatial-orientational correlation function $S^{110}(r)^{25}$

$$
S^{110}(r)=-\frac{1}{\sqrt{3}}\left\langle\delta\left(r-r_{12}\right)\left(\hat{\boldsymbol{z}}_{1} \cdot \hat{\boldsymbol{z}}_{2}\right)\right\rangle_{12},
$$

which is basically the average scalar product between the orientation of two molecules at distance $r$ and describes their tendency to align themselves parallel or antiparallel, as a function of separation.

In the case $\mu^{*}=1$ [Fig. 3(a)-(d)], $g_{0}(r)$ shows the characteristic double peak of a hexagonal arrangement of molecules in smectic layers; the strong layering is confirmed by the function $g(z)$, while $g\left(z_{\mathrm{d}}\right)$ indicates also a double stratification of dipoles 'up' and 'down' within each molecular layer (see also Fig. 2); finally the averaged Stone function $S^{110}(r)$ shows a weak correlation of antiparallel pairs. This indicates that the monolayer smectic is really of what we could call a $\mathrm{S}_{\mathrm{B}_{1}}$ type.

Increasing the dipole to $\mu^{*}=1.5$ [Figs. 4(a)-(d)] and also to $\mu^{*}=2$ [Figs. 5(a)-(d)], the $g_{0}(r)$ gives a more complicated

succession of peaks. At short range these small peaks are due to molecules which are not confined to the same plane as the molecule at the origin, but are also placed in the upper and lower neighbouring layers. Also the density $g(z)$ showing the splitting of the first peak and a spacing smaller than $\approx 2 \sigma_{\mathrm{s}}$, which is significantly less than the scaled molecular length $3 \sigma_{\mathrm{s}}$, is consistent with a structure where the layers are strongly interdigitated. The dipolar density $g\left(z_{\mathrm{d}}\right)$ presents the same periodicity and also indicates that dipoles tend to organise themselves in the interdigitation regions forming well defined antiferroelectric planes of closely packed dipoles (see also Fig. 2). Finally the $S^{110}(r)$ denotes the tendency for neighbouring dipoles to have parallel correlations.

\section{Theory}

We now turn to developing an approximate theory for the structure of highly ordered smectics of polar molecules. We wish to complement the simulation study, particularly examining the predicted effect of changing dipole position and strength.

We start by considering a system of $N$ polar molecules in a volume $V$ and at temperature $T$, interacting with the pair additive potential given in eqn. (4). Working in the NVT ensemble the equilibrium Helmholtz free energy per particle is approximated by extending the variational cluster expansion method $^{26}$ for describing spatially inhomogeneous ordered fluids. Retaining the lowest order of the approximation, the derived form of the free energy is a generalisation of the Onsager free energy and is appropriate to describe order- 
(a)

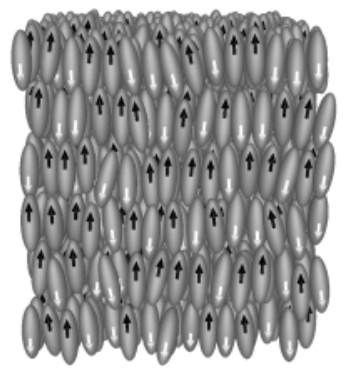

(c)

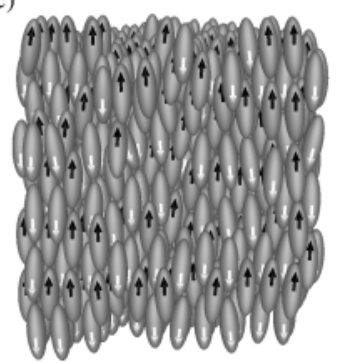

(e)

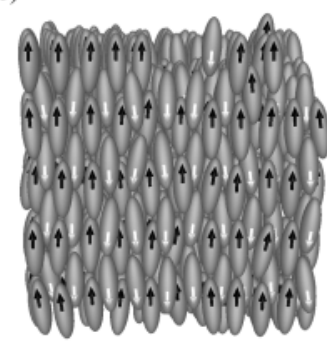

(b)

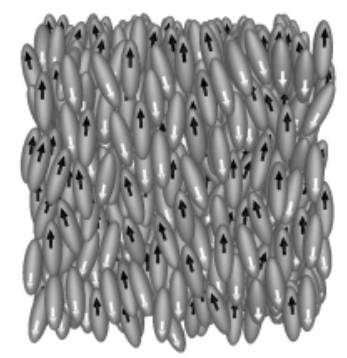

(d)

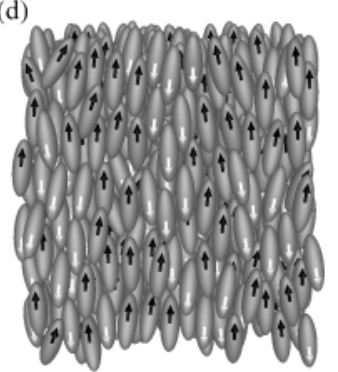

(f)

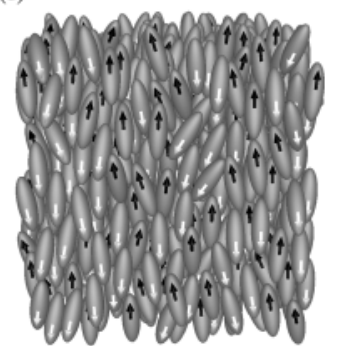

Fig. 2 Snapshots of the molecular organisation from the Monte Carlo simulations of the $N=1000$ system with an axial dipole: (a) $\mu^{*}=1$ and $T^{*}=1.2\left(\mathrm{~S}_{\mathrm{B}}\right) ;(\mathrm{b}) \mu^{*}=1$ and $T^{*}=1.8(\mathrm{~N}) ;(\mathrm{c}) \mu^{*}=1.5$ and $T^{*}=0.8$ $\left(\mathrm{S}_{\mathrm{A}_{\mathrm{d}}}\right) ;(\mathrm{d}) \mu^{*}=1.5$ and $T^{*}=1.2(\mathrm{~N}) ;(\mathrm{e}) \mu^{*}=2$ and $T^{*}=1.2\left(\mathrm{~S}_{\mathrm{A}_{\mathrm{d}}}\right) ;(\mathrm{f}) \mu^{*}=2$ and $T^{*}=2(\mathrm{~N})$.

disorder transitions (including isotropic, nematic and smectic A phase symmetries).

Let us assume that the system can exhibit one-dimensional long-range positional order, with periodicity $s_{\mathrm{d}}$, along the macroscopic $Z$ axis, while, in the $X Y$ plane, it behaves as a two-dimensional liquid. In this case the singlet distribution function (SDF) is of the form:

$$
P(\boldsymbol{r}, \hat{\boldsymbol{u}})=\frac{S_{\mathrm{d}}}{V} f(z, \boldsymbol{\omega}),
$$

with $f(z, \omega)=f\left(z \pm n s_{\mathrm{d}}, \omega\right), n=0,1,2, \ldots$, where $\omega$ is the orientation of $\hat{\boldsymbol{u}}$. According to the variational cluster approximation, the free energy density ${ }^{27,28}$ can be written as

$$
\frac{F}{N k_{\mathrm{B}} T}=\frac{F^{\text {ideal }}}{N k_{\mathrm{B}} T}-\frac{S}{N k_{\mathrm{B}}}+\frac{F^{\text {int }}}{N k_{\mathrm{B}} T},
$$

with

$$
\frac{F^{\text {ideal }}}{N k_{\mathrm{B}} T}=\ln \left(\frac{\rho \Lambda^{3}}{\Omega}\right)-1
$$

the ideal part, where $\Lambda=\Lambda_{\mathrm{t}} \Lambda_{\mathrm{r}}$ is the product of the translational and the orientational thermal wavelengths and $\Omega$ is the orientational 'volume'. The second, entropic, term corresponds to the loss of entropy if the SDF is not isotropic or spatially uniform:

$$
\frac{S}{N k_{\mathrm{B}}}=-\int_{0}^{s_{\mathrm{d}}} \mathrm{d} z \int \mathrm{d} \boldsymbol{\omega} f(z, \boldsymbol{\omega}) \ln \left[s_{\mathrm{d}} f(z, \boldsymbol{\omega})\right] .
$$

The last term in eqn. (11) corresponds to the interaction contributions

$$
\frac{F^{\mathrm{int}}}{N k_{\mathrm{B}} T}=\frac{1}{2} \rho s_{\mathrm{d}}\left\langle\left\langle q\left(z_{12}, \omega_{1}, \omega_{2}\right)\right\rangle\right\rangle_{f},
$$

where

$q\left(z_{12}, \boldsymbol{\omega}_{1}, \boldsymbol{\omega}_{2}\right)=\int_{-\infty}^{\infty} \mathrm{d} x_{12} \mathrm{~d} y_{12}\left\{1-\exp \left[-U\left(\boldsymbol{r}_{12}, \boldsymbol{\omega}_{1}, \boldsymbol{\omega}_{2}\right) / k_{\mathrm{B}} T\right]\right\}$
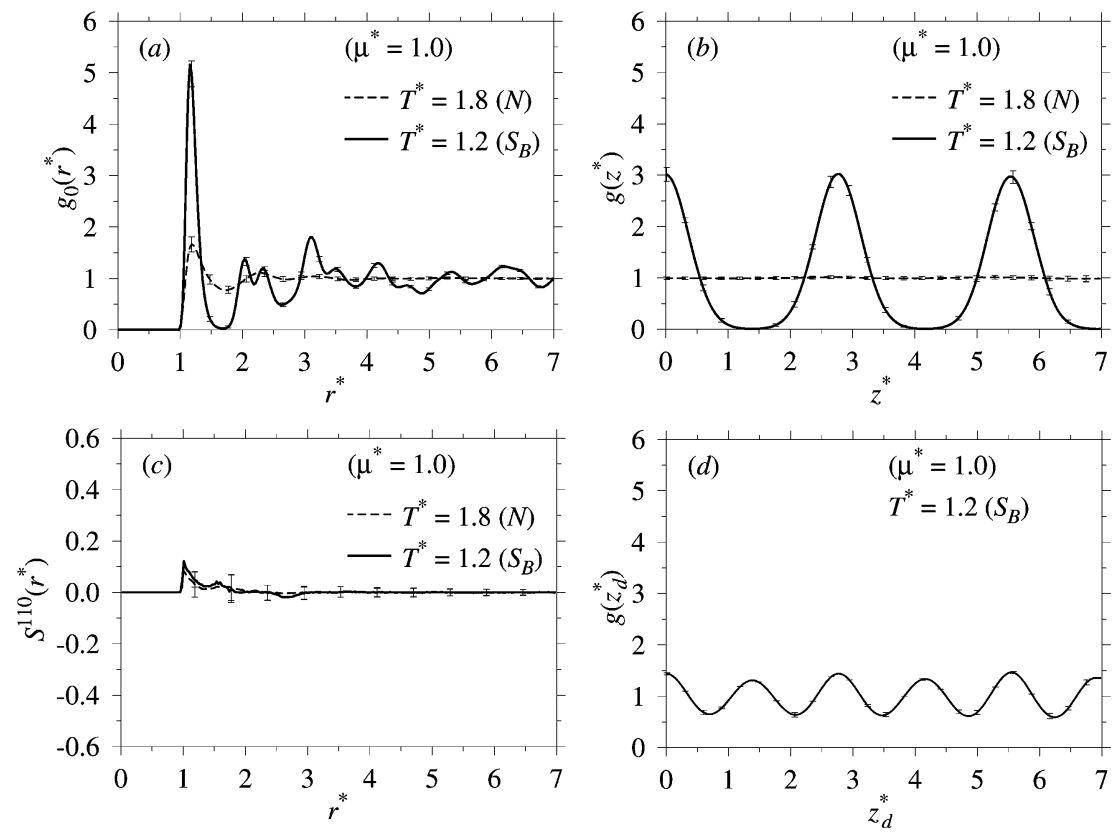

Fig. 3 Correlation functions from MC simulations: (a) radial $g_{0}\left(r^{*}\right)$, (b) density along the director $g\left(z^{*}\right)$, (c) orientational $S^{110}\left(r^{*}\right)$, and (d) dipolar density along the director $g\left(z_{\mathrm{d}}{ }^{*}\right)$ for the system of $N=1000$ rods with axial dipole $\mu^{*}=1$. The estimated fluctuations, shown here as error bars, are plotted each 10 sampling bins. 

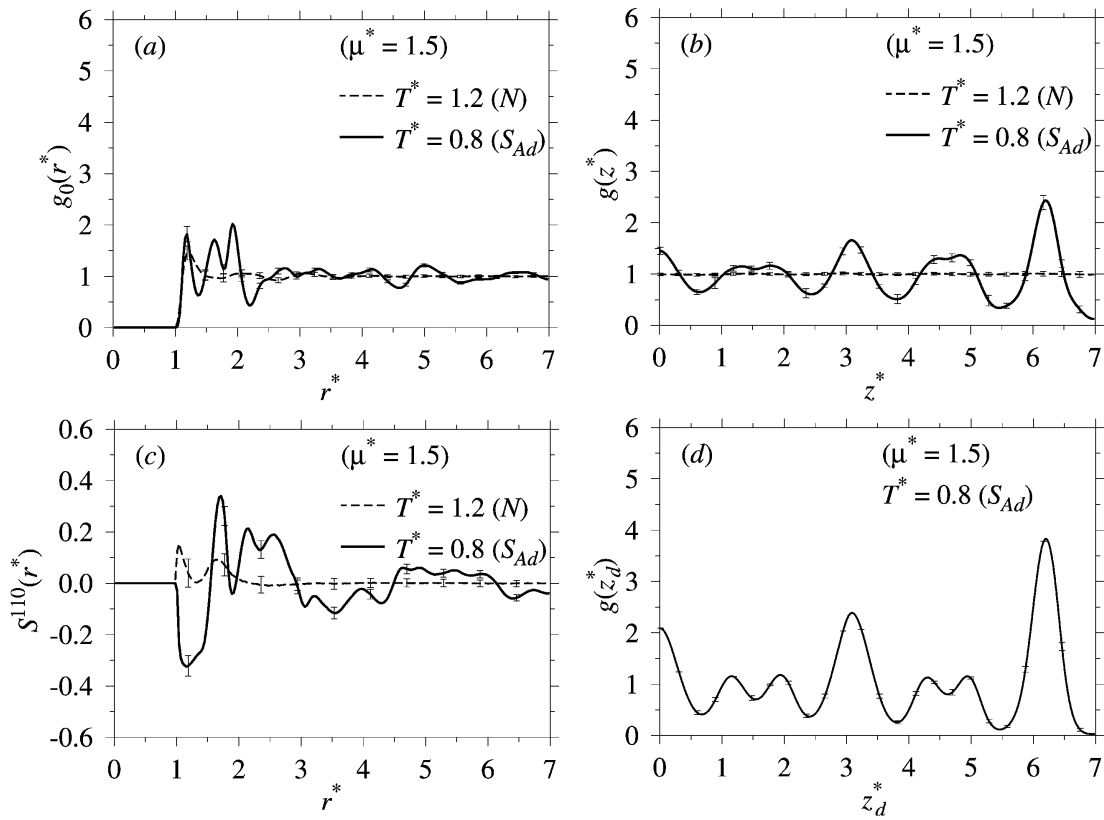

Fig. 4 Correlation functions from MC simulations: (a) radial $g_{0}\left(r^{*}\right)$, (b) density along the director $g\left(z^{*}\right)$, (c) orientational $S^{110}\left(r^{*}\right)$, and (d) dipolar density along the director $g\left(z_{\mathrm{d}}{ }^{*}\right)$ for the system of $N=1000$ rods with axial dipole $\mu^{*}=1.5$. The estimated fluctuations, shown here as error bars, are plotted each 10 sampling bins.

is the positional-orientational integral of the Mayer function of the intermolecular potential [eqn. (4)] and the double angle brackets indicate double averaging of the enclosed quantity with respect to the two singlet distributions $f\left(z_{1}, \omega_{1}\right)$ and $f\left(z_{2}\right.$, $\omega_{2}$ ). In the same approximation the one-particle distribution function is in turn given by

$$
f(z, \boldsymbol{\omega})=\zeta^{-1} \exp \left[-\rho s_{\mathrm{d}} \bar{q}(\mathrm{z}, \boldsymbol{\omega})\right]
$$

with

$$
\zeta=\int_{0}^{s_{\mathrm{d}}} \mathrm{d} z \int d \boldsymbol{\omega} \exp \left[-\rho s_{\mathrm{d}} \bar{q}(z, \boldsymbol{\omega})\right]
$$

and

$$
\bar{q}\left(z_{1}, \boldsymbol{\omega}_{1}\right)=\int_{-\infty}^{\infty} \mathrm{d} z_{2} \int \mathrm{d} \boldsymbol{\omega}_{2} q\left(z_{12}, \boldsymbol{\omega}_{1}, \boldsymbol{\omega}_{2}\right) f\left(z_{2}, \boldsymbol{\omega}_{2}\right) .
$$

Eqs. (16) and (18) constitute a set of self-consistency conditions from which, given $T, \rho$ and $s_{\mathrm{d}}$, the singlet distribution function is determined. Finally, for the layered phase, the requirement of minimisation of the free energy with respect to the layer spacing provides the equilibrium periodicity $s_{\mathrm{d}}$.

It is well known that molecular theories for hard particles with shape anisotropy can give, on the above level of approximation, only a qualitative description of the phase sequence. However, the resummation procedure of Parsons and Lee, ${ }^{29-31}$
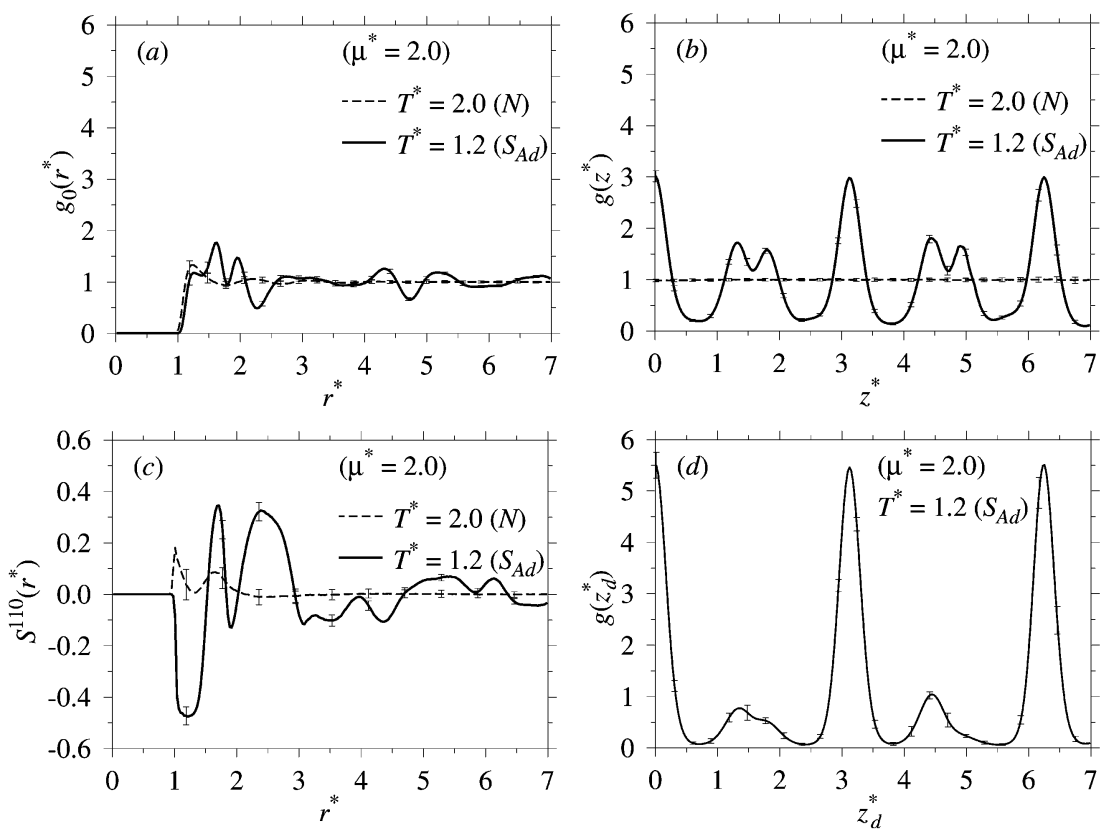

Fig. 5 Correlation functions from MC simulations: (a) radial $g_{0}\left(r^{*}\right)$, (b) density along the director $g\left(z^{*}\right)$, (c) orientational $S^{110}\left(r^{*}\right)$, and (d) dipolar density along the director $g\left(z_{\mathrm{d}}{ }^{*}\right)$ for the system of $N=1000$ rods with axial dipole $\mu^{*}=2$. The estimated fluctuations, shown here as error bars, are plotted each 10 sampling bins. 
with a simple rescaling of the number density, incorporates indirectly in the free energy all contributions due to molecular clusters of order higher than two. This procedure leads to a fairly good quantitative description of equations of state involving liquid-crystalline phases. Unfortunately, there are no analogous schemes for systems with particles interacting with 'soft' interactions.

Taking advantage of the separation of the intermolecular potential into a 'hard' and a 'soft' term, according to eqns. (4)-(6), we have

$$
q\left(z_{12}, \boldsymbol{\omega}_{1}, \boldsymbol{\omega}_{2}\right)=q_{0}\left(z_{12}, \boldsymbol{\omega}_{1}, \boldsymbol{\omega}_{2}\right)+q_{1}\left(z_{12}, \boldsymbol{\omega}_{1}, \boldsymbol{\omega}_{2}\right),
$$

with

$$
\begin{aligned}
& q_{0}\left.z_{12}, \boldsymbol{\omega}_{1}, \boldsymbol{\omega}_{2}\right) \\
& \quad=\int_{-\infty}^{\infty} \mathrm{d} x_{12} \mathrm{~d} y_{12}\left\{1-\exp \left[-U^{\text {hard }}\left(\boldsymbol{r}_{12}, \boldsymbol{\omega}_{1}, \boldsymbol{\omega}_{2}\right) / k_{\mathrm{B}} T\right]\right\},
\end{aligned}
$$

and

$$
\begin{aligned}
q_{1}\left(z_{12}, \boldsymbol{\omega}_{1}, \boldsymbol{\omega}_{2}\right)= & \int_{-\infty}^{\infty} \mathrm{d} x_{12} \mathrm{~d} y_{12} \exp \left[-U^{\text {hard }}\left(\boldsymbol{r}_{12}, \boldsymbol{\omega}_{1}, \boldsymbol{\omega}_{2}\right) / k_{\mathrm{B}} T\right] \\
& \times\left\{1-\exp \left[-U^{\text {soft }}\left(\boldsymbol{r}_{12}, \boldsymbol{\omega}_{1}, \boldsymbol{\omega}_{2}\right) / k_{\mathrm{B}} T\right]\right\} .
\end{aligned}
$$

Using eqn. (14) and assuming a standard resummation procedure (concerning the 'hard' part of the interaction potential) for the contributions to the free energy from particle clusters larger than two (i.e. Parsons-Lee approximation ${ }^{29-31}$ ), the free energy can be rewritten as

$$
\frac{F^{\text {int }}}{N k_{\mathrm{B}} T}=\frac{F^{\text {hard }}}{N k_{\mathrm{B}} T}+\frac{F^{\mathrm{soft}}}{N k_{\mathrm{B}} T},
$$

with

$$
\frac{F^{\text {hard }}}{N k_{\mathrm{B}} T}=\frac{1}{2} \rho s_{\mathrm{d}} a(\eta)\left\langle\left\langle q_{0}\left(z_{12}, \omega_{1}, \omega_{2}\right)\right\rangle\right\rangle_{f}
$$

and

$$
\frac{F^{\mathrm{soft}}}{N k_{\mathrm{B}} T}=\frac{1}{2} \rho s_{\mathrm{d}}\left\langle\left\langle q_{1}\left(z_{12}, \omega_{1}, \omega_{2}\right)\right\rangle\right\rangle_{f},
$$

and the singlet distribution function which now becomes

$$
f\left(z_{1}, \boldsymbol{\omega}_{1}\right)=\zeta^{-1} \exp \left\{-\rho s_{\mathrm{d}}\left[a(\eta) \bar{q}_{0}\left(z_{1}, \boldsymbol{\omega}_{1}\right)+\bar{q}_{1}\left(z_{1}, \boldsymbol{\omega}_{1}\right)\right]\right\},
$$

where we use the Parson-Lee approximation

$$
a(\eta)=\frac{(4-3 \eta)}{4(1-\eta)^{2}}
$$

instead of having $a(\eta)=1$, which corresponds to the simple two-molecule cluster approximation, with $\eta=N v_{0} / V, v_{0}$ the volume of the hard core of the particles.

Since we wish to study the nematic-smectic transition and the molecular organisation in the smectic phase, we make the assumption that the molecules are restricted to have their axes parallel. This assumption is consistent with our computer simulation results, according to which, in these smectics, the second-rank orientational order parameter takes quite high values, $\left\langle P_{2}\right\rangle \geqslant 0.9$. In this limit, integrals over orientations in the relations of this section are replaced by a summation over the two discrete orientations $w$, corresponding to the molecular dipole parallel $w_{+}$or antiparallel $w_{-}$to the macroscopic $Z$ axis:

$$
\int d \omega \Rightarrow \sum_{\left\{w_{+}, w_{-}\right\}} .
$$

The above restriction on the molecular orientations simplifies considerably the calculations of the positional-orientational functions of eqns. (20) and (21). Thus, $q_{0}\left(z_{12}, w_{1}, w_{2}\right)$ is readily calculated analytically and $q_{1}\left(z_{12}, w_{1}, w_{2}\right)$ by numerical integration (see the Appendix). Having the above kernels, the self-consistency condition in eqn. (25) is solved iteratively. ${ }^{32}$

All the above simplifications aim primarily at the development of a simple theory for studying the influence of molecular dipoles on the tendency of Gay-Berne particles to form smectic phases. Although the theory is applicable to first- as well as to second-order phase transitions, we do not attempt to address here the issue of the order of the $\mathrm{N}-\mathrm{S}_{\mathrm{A}}$ phase transition. Taking into consideration that the $\mathrm{N}-\mathrm{S}_{\mathrm{A}}$ phase transition is either second- or weak first-order, ${ }^{1}$ we carry out all the calculations at constant density, assuming that the volume change at the transition is negligible and does not affect the structural characteristics of the layered phases. Finally, in our calculations it is assumed, in keeping with the simulation results, that the systems under investigation do not exhibit macroscopic polarisation and therefore possible convergence problems of eqn. (24), due to the long-range character of dipolar interactions, are avoided. ${ }^{33,34}$

\subsection{Results from theory}

We find that for a system of purely repulsive hard Gaussian overlap ellipsoids, where the dipolar and attractive Gay-Berne interactions are absent, the nematic to smectic phase transition is exhibited at $\rho_{\mathrm{HGO}}{ }^{*} \approx 0.34$. When the attractive part of the GB potential is included, the smectic phase is stabilised with respect to the nematic, since the nematic to smectic transition moves to densities below $\rho_{\mathrm{HGO}}{ }^{*}$, for the whole range of temperatures.

It is known that using the Parsons-Lee resummation ${ }^{29-31}$ the transition densities of hard anisotropic systems are predicted fairly well. Thus we have chosen for the theoretical calculations the same density employed in the NVT MC simulations. Notwithstanding the good success of theory for the purely repulsive term, it is known that this type of theory strongly overestimates the transition temperature at given density. ${ }^{35}$ In an effort to make contact and compare with simulation results, this has led other workers ${ }^{35}$ to invoke different adjustable scaling factors for the strength of the average attractive contributions in the various phases (isotropic, nematic, smectic). Here we are interested in how the dipolar interactions affect the molecular organisation in smectics, and thus we simply compare theory and simulation using a reduced temperature scale $\left(T_{\mathrm{NS}}{ }^{*} / T_{0}{ }^{*}\right)$, at constant density $\rho^{*}=0.3$, as a function of the dipolar strength $\mu^{*}$ (Fig. 6). Here $T_{0}{ }^{*}$ is the temperature at which the transition for the apolar system takes place. The different curves correspond to theoretical results for various dipolar displacement, $d^{*}$, from the centre of the molecule. The symbols correspond to the scaled temperatures $\left(T_{\mathrm{NS}}{ }^{*(\mathrm{MC})} / T_{0}{ }^{*(\mathrm{MC})}\right)$ obtained from the MC simulations of the system with dipoles positioned at distance $d^{*}=0.65$ from the molecular centre, as described in the previous section.

As suggested by the plots of Fig. 6, the presence of a central dipole (continuous line) clearly moves the transition to higher temperatures and stabilises the smectic phase with respect to the nematic in agreement with previous simulation results in both Gay-Berne ${ }^{15}$ and hard spherocylinder systems. ${ }^{36}$ The situation changes upon displacing the dipole from the molecular centre: in this case the arrangement of the molecules in the usual $\mathrm{S}_{\mathrm{A}}$ layers does not correspond to the optimal dipolar energy. Moreover, increasing the dipole strength $\mu^{*}$ up to $\mu^{*} \approx 1.5$, the off-centre longitudinal dipole opposes the layering and destabilises the smectic phase with respect to the nematic, as we have also found from MC simulation, at least for $d^{*}=0.65$. Thus, for small and moderate dipolar strengths, the nematic-smectic transition takes place at a lower temperature upon moving the dipole from the centre near to the end of the molecule. For fairly strong dipoles $\left(\mu^{*} \geqslant 2\right)$ the layered phase gains some stability, probably in connection to bilayer formation. However, it should be noted that for strong dipoles 


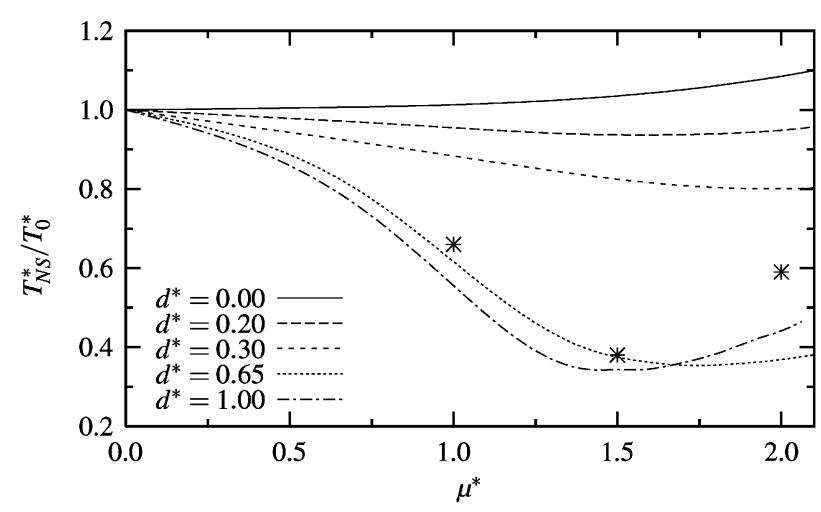

Fig. 6 Scaled nematic-smectic transition temperatures, at constant density $\rho^{*}=0.3$, as a function of the dipolar strength $\mu^{*}$, for different distances $d^{*}$ of the dipole from the centre of the molecule. The curves are results obtained from variational cluster theory. The values from the MC computer simulation with $d^{*}=0.65$ (stars) are reported for comparison.

the validity limits of the theory are reached since molecular association, due to dipolar dimerisation, is expected to play a crucial role in the system behaviour. ${ }^{37}$ Quite similarly, computer simulations where evolution takes place by standard single particle moves would potentially run into difficulties due to the dimerisation. In such cases cluster moves ${ }^{38}$ should probably be employed.

The layer spacing appears in general to be quite sensitive to both dipolar position and strength. Referring explicitly to the case of $\mathrm{d}^{*}=0.65$, studied in this paper, we see that the theoretical prediction for the nematic-smectic transition temperature is in fairly good agreement with the MC results. Indeed, the layer spacing for $\mu^{*}=1$ lies in the range 3.5 to $3.6 \sigma_{\mathrm{s}}$ (i.e. $\left.1.16-1.2 \sigma_{\mathrm{e}}\right)$, and is practically temperature-independent, indicating a well defined, not interdigitated, monolayer smectic structure. On increasing the dipolar strength, the layer spacing at the transition becomes wider and increases on lowering the temperature, going up to $4.5 \sigma_{\mathrm{s}}$ (i.e. $1.5 \sigma_{\mathrm{e}}$ ). At the same time, as can been seen in Fig. 7, the density profile within the layers becomes broader $\left(\mu^{*}=1.5\right)$ and for $\mu^{*}=2$ splits into two distinct sublayers indicating clearly a bilayer structure. In the latter case the distance between the two peaks approaches the separation distance $4.0 \sigma_{\mathrm{s}}$ (i.e. $1.3 \sigma_{\mathrm{e}}$ ) of the centres of two molecules with their dipoles dimerised in an antiparallel configuration.

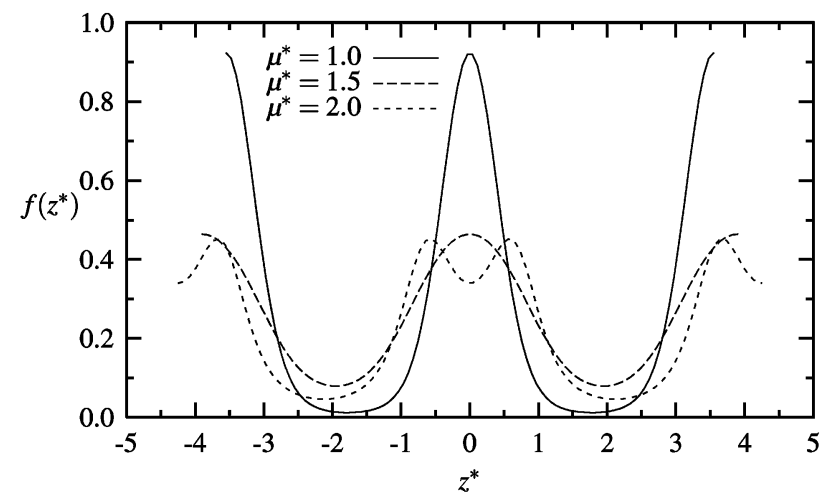

Fig. 7 Singlet distribution function at $T^{*}{ }_{\mathrm{NS}} / T^{*}{ }_{0}=0.33$ as obtained from variational cluster theory for the three different dipolar strengths and $d^{*}=0.65$.

\section{Conclusions}

Computer simulations and variational theory suggest that the overall molecular organisation of a smectic made of polar uniaxial mesogens and its dipole organisation are strongly influenced by the strength of the dipole when the latter is located at an intermediate position between the centre of the molecule and its end. At low temperatures a strongly interdigitated structure $S_{A_{d}}$, with an antiparallel arrangement of dipole moments, is formed and the degree of overlap between layers increases as the dipole strength becomes larger. Indeed, the origin of this structure may be due to the local coupling of the dipoles, which produces staggered configurations that cannot be packed into simple smectic monolayers.

\section{Appendix: Calculation of $q_{0}$ and $q_{1}$}

The excluded 'area' $q_{0}\left(z_{12}, w_{1}, w_{2}\right)$ of two parallel ellipsoids as a function of their centre-to-centre distance (the projection of the intermolecular vector on their common orientation), defined in eqn. (20), is easily calculated analytically:

$$
q_{0}\left(z_{12}, w_{1}, w_{2}\right)=\pi\left(\sigma_{\mathrm{s}}^{2}-\frac{z_{12}^{2}}{k^{2}}\right) \Theta\left(\sigma_{\mathrm{s}}^{2} k^{2}-z_{12}^{2}\right),
$$

where $\Theta$ is the Heaviside step function and $k=\sigma_{\mathrm{e}} / \sigma_{\mathrm{s}}$. Obviously, there is no dependence on the orientation of the molecules. To handle the long-range character of the dipolar interactions, we separate the calculation of $q_{1}\left(z_{12}, w_{1}, w_{2}\right)$, defined in eqn. (21), into two parts:

(a) The short-range part $q_{1}^{\mathrm{s}}\left(z_{12}, w_{1}, w_{2}\right)$, which is calculated numerically. This part receives contributions only from configurations for which the second molecule lies within a cutoff cylinder centred at the first one. Obviously for these configurations the Gay-Berne potential is non-zero, $U^{\mathrm{GB}} \neq 0$. Performing the integrations in cylindrical coordinates we have

$$
\begin{aligned}
& q_{1}^{\mathrm{s}}\left(z_{12}, w_{1}, w_{2}\right) \\
& \quad=2 \pi \int_{0}^{R_{c}} \mathrm{~d} \rho_{12} \rho_{12}\left\{1-\exp \left[-\frac{1}{k_{\mathrm{B}} T} U^{\mathrm{soft}}\left(\boldsymbol{r}_{12}, w_{1}, w_{2}\right)\right]\right\},
\end{aligned}
$$

where the radius of the cylinder $R_{\mathrm{c}}$ is the cutoff distance for the Gay-Berne potential $R_{\mathrm{c}}{ }^{\mathrm{GB}}$ and $\left|z_{12}\right|<R_{\mathrm{c}}{ }^{\mathrm{GB}}$.

(b) The long-range part $q_{1}^{l}\left(z_{12}, w_{1}, w_{2}\right)$, to which only the configurations with the second molecule positioned outside the cutoff cylinder contribute. For such configurations $U^{\mathrm{GB}}=0$. The large intermolecular distance allows the expansion of the term $\left[1-\exp \left[-U^{\mathrm{d}}\left(\boldsymbol{r}_{12}, w_{1}, w_{2}\right) /\left(k_{\mathrm{B}} T\right)\right]\right]$ in powers of $\mu^{2} /$ $\left(r_{\mathrm{d}}^{3} k_{\mathrm{B}} T\right)$ and we get

$$
\begin{aligned}
q_{1}^{l}\left(z_{12}, w_{1}, w_{2}\right)= & 2 \pi s_{\mathrm{d}} \int_{R_{c}}^{\infty} \mathrm{d} \mathfrak{J}_{12} \mathfrak{J}_{12} \\
& \sum_{m=1} \frac{1}{m !}\left[-\frac{1}{k_{\mathrm{B}} T} U^{\mathrm{d}}\left(\boldsymbol{r}_{12}, w_{1}, w_{2}\right)\right]^{m},
\end{aligned}
$$

where $R_{\mathrm{c}}=0$ if $\left|z_{12}\right|>R_{\mathrm{c}}{ }^{\mathrm{GB}}$ and $R_{\mathrm{c}}=R_{\mathrm{c}}{ }^{\mathrm{GB}}$ if $\left|z_{12}\right| \leqslant R_{\mathrm{c}}{ }^{\mathrm{GB}}$. In the above expansion each term can be calculated analytically. In our calculations we have truncated the expansion past the $m=6$ term.

Having obtained the functions $q_{0}\left(z_{12}, w_{1}, w_{2}\right)$ and $q_{1}\left(z_{12}\right.$, $\left.w_{1}, w_{2}\right)$ we can proceed to calculate the SDF at a given $T$ and $\rho$ for various periodicities $s_{\mathrm{d}}$. This is done numerically using the following iterative scheme: ${ }^{32}$

$$
\begin{aligned}
f_{m+1}\left(z_{1}, w_{1}\right)= & \zeta^{-1} \exp \left\{-\rho s_{\mathrm{d}}\left[a(\eta)\left\langle q_{0}\left(z_{12}, w_{1}, w_{2}\right)\right\rangle_{f_{m}\left(z_{2}, w_{2}\right)}\right.\right. \\
& \left.\left.+\left\langle q_{1}\left(z_{12}, w_{1}, w_{2}\right)\right\rangle_{f_{m}\left(z_{2}, w_{2}\right)}\right]\right\},
\end{aligned}
$$


with convergence criterion $\left|f_{m+1}\left(z_{1}, w_{1}\right)-f_{\mathrm{m}}\left(z_{1}, w_{1}\right)\right|<10^{-5} \sigma_{\mathrm{s}}{ }^{-1}$, for all $\left(z_{1}, w_{1}\right)$. Here the angle brackets indicate averaging with respect to the singlet distribution $f_{m}\left(z_{2}, w_{2}\right)$.

\section{Acknowledgements}

We are grateful to EU TMR (contract FMRX-CT97-0121) for support and fellowship for A. Vanakaras and S. Orlandi. We also thank the University of Bologna, MURST PRIN 'Cristalli Liquidi' and CNR PF-MSTA2 for financial support towards the computer resources employed.

\section{References}

1 P. G. de Gennes and J. Prost, The Physics of Liquid Crystals, 2nd edn., Oxford University Press, Oxford, 1993.

2 G. W. Gray and J. W. Goodby, Smectic Liquid Crystals, Textures And Structures, Leonard Hill, Glasgow, 1984.

3 M. F. Achard, F. Hardouin, A. M. Levelut and G. Sigaud, J. Chem. Phys., 1983, 80, 537.

4 A. M. Levelut, R. J. Tarento, F. Hardouin, M. F. Achard and G. Sigaud, Phys. Rev. A, 1981, 24, 2180.

5 J. O. Indekeu and A. N. Berker, J. Phys. (Paris), 1983, 80, 65.

6 B. I. Ostrovsky, Liq. Cryst., 1993, 14, 131.

7 J. Prost and P. Barois, J. Chim. Phys., 1980, 80, 65.

8 L. Longa and W. de Jeu, Phys. Rev. A, 1983, 28, 2380.

9 G. Sigaud, F. Hardouin, M. F. Achard and A. M. Levelut, J. Phys. (Paris), 1981, 42, 107.

10 J. J. Weis, D. Levesque and G. J. Zarragoicoechea, Phys. Rev. E, 1993, 47, 496.

11 J. J. Weis, D. Levesque and G. J. Zarragoicoechea, Phys. Rev. Lett., 1992, 69, 913.

12 R. Berardi, S. Orlandi and C. Zannoni, Chem. Phys. Lett., 1996, 261, 357.

13 S. Mita, K. Satoh and S. Kondo, Liq. Cryst., 1996, 20, 757.
14 S. Mita, K. Satoh and S. Kondo, Chem. Phys. Lett., 1996, 255, 99.

15 M. Houssa, L. F. Rull and S. C. McGrother, J. Chem. Phys., 1998, 109, 9529.

16 J. Gay and B. J. Berne, J. Chem. Phys., 1981, 74, 3316.

17 R. Berardi, A. P. J. Emerson and C. Zannoni, J. Chem. Soc., Faraday Trans., 1993, 89, 4069.

18 J. A. Barker and R. O. Watts, Mol. Phys., 1973, 26, 789.

19 R. Berardi, S. Orlandi and C. Zannoni, Int. J. Mod. Phys. C, 1999, 10, 477.

20 A. Gil-Vilegas, S. C. McGrother and G. Jackson, Mol. Phys., 1997, 92, 723.

21 M. Houssa, A. Oualid and L. F. Rull, Mol. Phys., 1998, 94, 439.

22 C. Zannoni, in The Molecular Physics of Liquid Crystals, ed. G. R. Luckhurst and G. W. Gray, Academic Press, London, 1979, ch. 3.

23 U. Fabbri and C. Zannoni, Mol. Phys., 1986, 58, 763.

24 C. Zannoni, in Advances in the Computer Simulations of Liquid Crystals, ed. P. Pasini and C. Zannoni, Kluwer, Dordrecht, 2000, ch. 2.

25 A. J. Stone, Mol. Phys., 1978, 36, 241.

26 A. G. Vanakaras and D. J. Photinos, Mol. Phys., 1995, 85, 1089.

27 C. G. Gray and K. E. Gubbins, Theory of Molecular Fluids, Oxford University Press, Oxford, 1984.

28 M. P. Allen, G. T. Evans, D. Frenkel and B. M. Mulder, in Advanced Materials, vol. 86, ed. I. Prigogine and S. A. Rice, John Wiley and Sons, New York, 1993.

29 J. D. Parson, Phys. Rev. A, 1979, 19, 1225.

30 S. D. Lee, J. Chem. Phys., 1987, 87, 4972.

31 S. D. Lee, J. Chem. Phys., 1989, 89, 7036.

32 J. Herzfeld, A. E. Berger and J. W. Wingate, Macromolecules, 1984, 17, 1718

33 M. A. Osipov, P. I. C. Teixeira and M. M. Telo da Gama, J. Phys. A: Math. Gen., 1997, 30, 1953.

34 S. Banerjee, R. B. Griffiths and M. Widom, J. Stat. Phys., 1998, 93, 109.

35 E. Velasco and L. Mederos, J. Chem. Phys., 1998, 109, 2361.

36 S. C. McGrother, A. Gil-Vilegas and G. Jackson, Mol. Phys., 1998, 95, 657.

37 A. V. Emelyanenko and M. A. Osipov, Liq. Cryst., 1999, 26, 187.

38 R. Berardi, M. Fehervari and C. Zannoni, Mol. Phys., 1999, 97, 1173. 